\title{
Effect of endoscopy screening on stage at gastric cancer diagnosis: results of the National Cancer Screening Programme in Korea
}

\begin{abstract}
K S Choi ${ }^{1,2}$, J K Jun *,1,2, M Suh ${ }^{1}$, B Park ${ }^{1}$, D K Noh ${ }^{1}$, S H Song ${ }^{1}$, K W Jung ${ }^{1}$, H-Y Lee ${ }^{3}$, I J Choi ${ }^{4}$ and E-C Park ${ }^{5}$
${ }^{1}$ National Cancer Control Institute, National Cancer Centre, 323, Ilsan-ro, Ilsandong-gu, Goyang-si, Gyeonggi-do 410-769, Republic of Korea; ${ }^{2}$ Graduate School of Cancer Science and Policy, National Cancer Centre, 323, Ilsan-ro, Ilsandong-gu, Goyang-si, Gyeonggi-do 410-769, Republic of Korea; ${ }^{3}$ Department of Social Medicine, College of Medicine, Dankook University, 119, Dandae-ro, Dongnam-gu, Cheonan-si, Chungnam 330-714, Republic of Korea; ${ }^{4}$ Gastric Cancer Branch, Research Institute and Hospital, National Cancer Center, 323, Ilsan-ro, Ilsandong-gu, Goyang-si, Gyeonggi-do 410-769, Republic of Korea and ${ }^{5}$ Department of Preventive Medicine and Institute of Health Services Research, College of Medicine, Yonsei University, 50-1, Yonsei-ro, Seodaemun-gu, Seoul 120-752, Republic of Korea
\end{abstract}

Background: Although gastric cancer screening is common among countries with a high prevalence of gastric cancer, there is little data to support the effectiveness of this screening. This study was designed to determine the differences in stage at diagnosis of gastric cancer according to the screening history and screening method (upper gastrointestinal series (UGIS) vs endoscopy).

Methods: The study population was derived from the National Cancer Screening Programme (NCSP), a nationwide organised screening programme in Korea. The study cohort consisted of 19168 gastric cancer patients who had been diagnosed in 2007 and who were invited to undergo gastric cancer screening via the NCSP between 2002 and 2007.

Results: Compared with never-screened patients, the odds ratios for being diagnosed with localised gastric cancer in endoscopyscreened patients and UGIS-screened patients were $2.10(95 \% \mathrm{Cl}=1.90-2.33)$ and $1.24(95 \% \mathrm{Cl}=1.13-1.36)$, respectively.

Conclusions: Screening by endoscopy was more strongly associated with a diagnosis of localised stage gastric cancer compared with screening by UGIS.

Although, the incidence of gastric cancer is declining worldwide, gastric cancer remains the third most common cause of cancer death (Ferlay et al, 2013). Survival from gastric cancer remains low in the Western world, with reported 5-year survival rates of 10$30 \%$ (Karim-Kos et al, 2008), in contrast to survival rates in Asia of 69\% (Nashimoto et al, 2013; Jung et al, 2014). The higher survival rate from gastric cancer is partly attributed to the availability of screening programmes. In countries with a high prevalence of gastric cancer, specifically in Japan and Korea, gastric cancer screening is commonly conducted.

Japan has been conducting mass gastric cancer screening using photofluorography (via indirect upper gastrointestinal series (UGIS)) since 1960 and has achieved a remarkable reduction in mortality rates and consequently higher cure rates (Fukao et al, 1995; Mizoue et al, 2003; Miyamoto et al, 2007). In recent years, the main method used for opportunistic screening in Asia has been

*Correspondence: Dr JK Jun; E-mail: jkjun@ncc.re.kr

Received 2 June 2014; revised 27 October 2014; accepted 11 November 2014;

published online 9 December 2014

(c) 2015 Cancer Research UK. All rights reserved 0007-0920/15

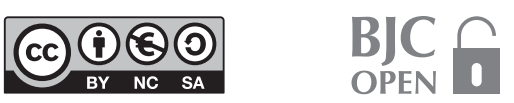


endoscopy (Leung et al, 2008). In Korea, a nationwide gastric cancer screening programme was started in 1999 as part of the National Cancer Screening Programme (NCSP). The NCSP provides biennial gastric cancer screening for men and women aged 40 or over, by either UGIS or endoscopy (Kim et al., 2011). Recently, a case-control study and a cohort study in Japan reported a $30-65 \%$ reduction in gastric cancer mortality when screening was undertaken via endoscopy (Hosokawa et al, 2008; Hamashima et al, 2013). However, there is a lack of agreement about which method is most effective in the general population.

Although randomised controlled trials represent the most reliable method for evaluating the impact of screening on cancer mortality reduction, such intervention studies are not feasible in most countries because gastric screening is already being widely conducted. Thus, no randomised control trial addressing the efficacy of gastric cancer screening on mortality reduction has been published. Furthermore, obtaining accurate estimates of reductions in mortality requires long-term follow-up of large populations. Therefore, stage at diagnosis was used as an intermediate outcome measure, as it is the most powerful predictor of patient survival. To date, no study has reported differences in stage at diagnosis of gastric cancer among gastric cancer patients who have undergone endoscopy screening and those who have undergone UGIS screening. Thus, the primary aim of this study was to determine whether differences in stage at diagnosis of gastric cancer exist among gastric cancer patients who participated in the NCSP (ever screened) and those who have no screening history (never screened). Furthermore, this study aimed to evaluate whether there is an association between stage at diagnosis of gastric cancer and screening method (UGIS $v s$ endoscopy).

\section{MATERIALS AND METHODS}

The study cohort consisted of patients aged 40 years or over with a diagnosis of gastric cancer reported in the Korean Central Cancer Registry (KCCR) in 2007. Among the 25915 gastric cancer patients identified, 19168 had been invited to gastric cancer screening through the NCSP in 2002-2007 and were included in the final analysis. In the NCSP, all men and women aged 40 or over (no upper age limit) receive an invitation letter from the National Health Insurance Service (NHIS) at the beginning of the year. Individuals invited to participate in the NCSP for gastric cancer can choose to undergo either UGIS or endoscopy screening at a clinic or hospital designated as a gastric cancer screening unit by the NHIS. Participants with a positive screening result on UGIS are offered a follow-up endoscopy test within the framework of the NCSP. The screening units report all screening results to the NHIS through a web-based database maintained by the NHIS (Kim et al, 2011).

Using the NCSP database, gastric cancer patients were classified by screening history in 2002-2007 (9191 never screened and 9977 ever screened). Never-screened gastric cancer patients were matched with ever-screened gastric cancer patients according to age, gender, and socioeconomic status. If there was no age match for a given patient, we considered those within the range of 2 years above or below the target age. A total of 8044 never-screened gastric cancer patients were matched to the same number of everscreened gastric cancer patients. We obtained written informed consent form participants who underwent screening for the collection of their screening results and health data recorded during the screening process. This retrospective study was approved by the institutional review board of the National Cancer Center, Korea.

Information on stage at gastric cancer diagnosis, anatomic site, and histological classification was obtained from the KCCR.
Tumour stage was recorded as localised, regional, distant, or unknown, in accordance with the categories used in the Surveillance, Epidemiology, and End Results (SEER) Cancer Statistics Review of the National Cancer Institute (Young et al, 2001). Localised neoplasms are confined entirely to the stomach and lack serosal involvement; intramucosal tumours are also classified as localised cancer. Regional cancers comprise tumours limited to the gastric wall with regional lymph node involvement or tumours with extension to nearby viscera. Distant neoplasms exhibit distant extension or metastases in the viscera or distant lymph nodes. Unknown stage refers to neoplasms lacking sufficient or unavailable information with which to assign a category. Information on socioeconomic status and screening history was extracted from the NCSP database. Gastric cancer patients were categorised by screening method (UGIS, endoscopy, and never screened), screening frequency (number of screenings received between 2002 and 2007: once, twice, three times or more, or never screened), and interval between date of gastric cancer diagnosis and the preceding screening date. The demographic characteristics of case and controls were compared using $\chi^{2}$-tests.

Conditional logistic regression was performed either including or excluding 'unknown stage' to calculate odds ratios (ORs) and 95\% confidence intervals (CIs) for being diagnosed with localised gastric cancer. We conducted subgroup analyses stratified by age, sex, socioeconomic status, screening method, screening frequency, and time interval since screening. The SAS software package (ver. 9.1; SAS Institute Inc., Cary, NC, USA) was used for all statistical calculations.

\section{RESULTS}

Baseline demographic and tumour characteristics of ever-screened and never-screened gastric cancer patients are shown in Table 1. Noncardia gastric cancer, intestinal histological subtypes, and localised gastric cancer were significantly more frequent in everscreened patients compared with never-screened patients $(P<0.001)$.

Figure 1 shows the distribution of stage at gastric cancer diagnosis. Localised gastric cancer was most frequently noted in both never-screened and ever-screened patients; however, between these two groups, localised cancer were more frequently recorded in ever-screened patients (Figure 1A). In addition, localised cancers were most frequent in patients who had undergone endoscopy, compared with patients who had undergone UGIS or neverscreened patients (Figure 1B).

We evaluated the odds of being diagnosed with localised gastric cancer $v$ s regional, distant, or unknown-stage gastric cancer in each subgroup according to screening history (Table 2). Overall, everscreened patients were statistically significantly more likely to be diagnosed with localised gastric cancer (adjusted OR $(\mathrm{aOR})=1.71$; $95 \% \mathrm{CI}=1.60-1.82)$. This odds increased when 'unknown stage' was removed from the analysis $(\mathrm{aOR}=1.81 ; 95 \% \mathrm{CI}=1.68-1.96)$. Compared with the never-screened patients, patients who had undergone screening by endoscopy were also more likely to be diagnosed with localised gastric cancer ( $\mathrm{aOR}=2.10 ; 95 \%$ $\mathrm{CI}=1.90-2.33)$. Although the risk of localised gastric cancer increased by 1.24 among patients screened by UGIS, compared with never-screened patients, the OR was much lower than that for patients screened by endoscopy. Further, patients who had undergone screening by endoscopy were 1.71 times (95\% $\mathrm{CI}=1.55-1.89)$ more likely to be diagnosed with localised gastric cancer compared with UGIS-screened patients (not shown in table). The OR for being diagnosed with localised gastric cancer was highest among patients who had a screening frequency of three times or more. Compared with never-screened patients, the OR for 
Table 1. Demographic and tumour characteristics of everscreened and never-screened gastric cancer patients, National Cancer Screening Programme

\begin{tabular}{|c|c|c|c|}
\hline Characteristics & $\begin{array}{c}\text { Never } \\
(n=8044) \\
n(\%)\end{array}$ & $\begin{array}{c}\text { Ever } \\
(n=8044) \\
n(\%)\end{array}$ & $P$-value \\
\hline Gender & & & 1.000 \\
\hline Male & $5685(70.7)$ & $5685(70.7)$ & \\
\hline Female & $2359(29.3)$ & $2359(29.3)$ & \\
\hline Age at diagnosis, years & & & 0.854 \\
\hline$\leqslant 49$ & $839(10.4)$ & 807 (10.3) & \\
\hline $50-59$ & $2059(25.6)$ & $2080(25.9)$ & \\
\hline $60-69$ & 2495 (31.0) & $2490(31.0)$ & \\
\hline $70 \leqslant$ & $2651(33.0)$ & $2667(33.2)$ & \\
\hline Socioeconomic status & & & 1.000 \\
\hline $\mathrm{NHI}$ with premium under $50 \%$ & $3729(46.4)$ & $3729(46.4)$ & \\
\hline $\mathrm{NHI}$ with premium over $50 \%$ & $3823(47.5)$ & $3823(47.5)$ & \\
\hline MAP recipients & $492(6.1)$ & $492(6.1)$ & \\
\hline Anatomic site & & & $<0.001$ \\
\hline Noncardia & $5852(72.8)$ & $6150(76.5)$ & \\
\hline Cardia & $320(4.0)$ & $331(4.1)$ & \\
\hline Overlapping & $722(9.0)$ & $593(7.4)$ & \\
\hline Unspecified & $1150(14.3)$ & $970(12.1)$ & \\
\hline Histological subtype & & & $<0.001$ \\
\hline Intestinal & $3941(49.0)$ & $4446(55.3)$ & \\
\hline Diffuse & $1336(16.6)$ & $1294(16.1)$ & \\
\hline Other or unspecified & $2767(34.4)$ & $2304(28.6)$ & \\
\hline Stage $^{a}$ & & & $<0.001$ \\
\hline Localised & $3264(40.6)$ & $4326(53.8)$ & \\
\hline Regional & $2372(29.5)$ & 1905 (23.7) & \\
\hline Distant & $1134(14.1)$ & $660(8.2)$ & \\
\hline Unknown & $1274(15.8)$ & $1153(14.3)$ & \\
\hline \multicolumn{4}{|c|}{$\begin{array}{l}\text { Abbreviations: MAP = medical aids programme; } \mathrm{NHI}=\text { national health insurance; SEER = } \\
\text { Surveillance, Epidemiology, and End Results; UGIS = upper gastrointestinal series. } \\
{ }^{\text {a }} \text { Stage definitions adapted from the SEER Cancer Statistics Review were applied: localised, } \\
\text { a neoplasm confined entirely to the stomach without serosal involvement; regional, a } \\
\text { neoplasm that extends beyond the limits of the stomach and invades the surrounding } \\
\text { tissue; distant, a neoplasm that spreads to parts of the body remote from the primary } \\
\text { tumour; and unknown, a neoplasm with insufficient or unavailable information to assign a } \\
\text { stage. }\end{array}$} \\
\hline
\end{tabular}

being diagnosed with localised gastric cancer was the highest within 12 months of diagnosis $(\mathrm{aOR}=1.82 ; 95 \% \mathrm{CI}=1.69-1.96)$, after which the ORs tended to decrease with an increasing time interval.

\section{DISCUSSION}

In the current study, we noted a large and highly significant increase in the diagnosis of localised gastric cancer, and a corresponding decrease in the diagnosis of regional and distal gastric cancer, among ever-screened patients relative to neverscreened patients. As well, endoscopy was more strongly associated with an earlier stage of gastric cancer at diagnosis than UGIS.

As stage at diagnosis of gastric cancer is well correlated with survival rate, (Jung et al, 2013) in the current study, we assumed that early detection of gastric cancer by screening may increase patient survival. A previous study conducted on patients with cardia adenocarcinoma found that $49 \%$ of gastric cancer patients who underwent previous endoscopy presented with in situ or localised gastric cancer, compared with $27 \%$ of those who did not have a previous endoscopy (Cooper et al, 2002). These findings are compatible with our study and are consistent with the notion that screening by endoscopy leads to earlier diagnosis of gastric cancer.
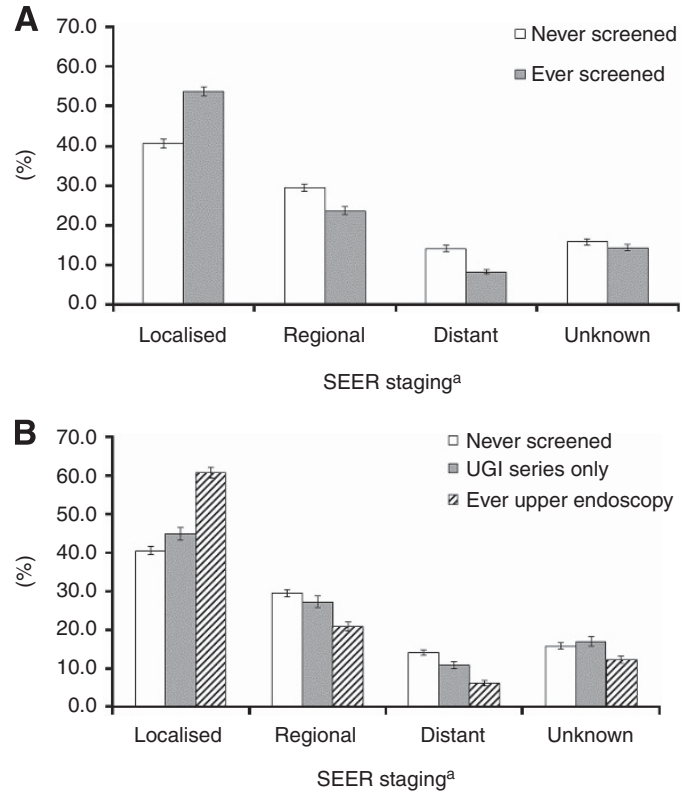

Figure 1. (A) Distribution of stage at gastric cancer diagnosis in patients according to history of gastric cancer screening via the National Cancer Screening Programme. (B) Distribution of gastric cancer stages in

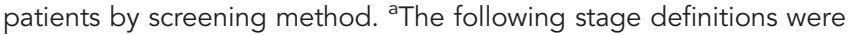
applied (adapted from the SEER Cancer Statistics Review): localised, a neoplasm confined entirely to the stomach without serosal involvement; regional, a neoplasm that extends beyond the limits of the stomach and invades the surrounding tissue; distant, a neoplasm that spreads to parts of the body remote from the primary tumour; and unknown, a neoplasm with insufficient or unavailable information to assign a stage.

In the current study, patients screened by endoscopy were 2.1 times more likely to be diagnosed with localised gastric cancer. Although the odds of having localised gastric cancer also increased by 1.24 among patients screened by UGIS, the odds were much lower than that for patients screened by endoscopy. Thus, we could suggest that endoscopy is more likely to detect localised gastric cancer than UGIS.

We also evaluated who might benefit from gastric cancer screening according to time interval since last screening. Compared with never-screened patients, the ORs were highest for those screened within 12 months of diagnosis and tended to decrease with an increasing time interval. A few Asian studies have reported that screening by endoscopy every 2 years increases the detection rate of early-stage gastric cancer (Mori et al, 2001; Nam et al, 2009). A cohort study conducted in Korea also reported that the risk of higher cancer stage at diagnosis increased by $23 \%$ per increase in 1 year interval length $(\mathrm{OR}=1.23 ; 95 \% \mathrm{CI}=1.19-1.28)$, and endoscopy intervals of 3 years or less showed similar benefits (Nam et al, 2012).

This study had several limitations. First, in order to evaluate the effects of cancer screening, study subjects should be asymptomatic; however, it was not possible to exclude symptomatic individuals in the NCSP database. As symptomatic individuals are more likely to be diagnosed with advanced-stage gastric cancer, the proportion of early-stage diagnoses in the ever-screened group might be underestimated. Second, screening history outside of the NCSP was unclear. Screening history was identified based on the participant lists for gastric cancer screening from 2002 to 2007 through the NCSP database. However, opportunistic screening for gastric cancer using endoscopy is often performed in Korea. Thus, among the never-screened group, there may have been some individuals who had undergone opportunistic gastric cancer screening. 
Table 2. ORs and $95 \% \mathrm{Cls}$ for the detection of localised gastric cancer for ever-screened patients compared with never-screened patients ${ }^{a}$

\begin{tabular}{|c|c|c|c|c|}
\hline & \multicolumn{2}{|c|}{\begin{tabular}{|c|} 
Including \\
'unknown stage'
\end{tabular}} & \multicolumn{2}{|c|}{$\begin{array}{c}\text { Excluding } \\
\text { 'unknown stage' }\end{array}$} \\
\hline & OR & $(95 \% \mathrm{Cl})$ & OR & $(95 \% \mathrm{Cl})$ \\
\hline Overall & 1.71 & $(1.60-1.82)$ & 1.81 & $(1.68-1.96)$ \\
\hline \multicolumn{5}{|l|}{ Gender $^{b}$} \\
\hline Male & 1.74 & $(1.62-1.88)$ & 1.84 & $(1.68-2.01)$ \\
\hline Female & 1.64 & $(1.45-1.84)$ & 1.76 & $(1.53-2.02)$ \\
\hline \multicolumn{5}{|l|}{ Age at diagnosis, years ${ }^{b}$} \\
\hline$\leqslant 49$ & 1.91 & $(1.56-2.35)$ & 1.97 & $(1.56-2.49)$ \\
\hline $50-59$ & 1.80 & $(1.58-2.04)$ & 2.02 & $(1.74-2.34)$ \\
\hline $60-69$ & 1.85 & $(1.65-2.07)$ & 1.92 & $(1.68-2.19)$ \\
\hline $70 \leqslant$ & 1.47 & $(1.32-1.64)$ & 1.51 & $(1.32-1.73)$ \\
\hline \multicolumn{5}{|l|}{ Socioeconomic status ${ }^{b}$} \\
\hline $\mathrm{NHI}$ with premium under $50 \%$ & 1.48 & $(1.35-1.62)$ & 1.63 & $(1.46-1.82)$ \\
\hline $\mathrm{NHI}$ with premium over $50 \%$ & 1.95 & $(1.77-2.14)$ & 2.03 & $(1.82-2.26)$ \\
\hline MAP recipients & 1.95 & $(1.50-2.55)$ & 1.73 & $(1.24-2.41)$ \\
\hline \multicolumn{5}{|l|}{ Screening method } \\
\hline Never-screened & 1.00 & Reference & 1.00 & Reference \\
\hline UGIS & 1.24 & $(1.13-1.36)$ & 1.28 & $(1.14-1.43)$ \\
\hline Endoscopy & 2.10 & $(1.90-2.33)$ & 2.31 & $(2.04-2.60)$ \\
\hline Both modalitieis, alternatively & 2.63 & $(2.20-3.15)$ & 2.83 & $(2.29-3.51)$ \\
\hline \multicolumn{5}{|l|}{ Screening frequency } \\
\hline Never screened & 1.00 & Reference & 1.00 & Reference \\
\hline Once & 1.51 & (1.39-1.63) & 1.59 & $(1.45-1.75)$ \\
\hline Twice & 2.00 & $(1.76-2.27)$ & 2.16 & $(1.86-2.51)$ \\
\hline Three times or more & 2.78 & $(2.22-3.49)$ & 2.91 & $(2.22-3.82)$ \\
\hline \multicolumn{5}{|c|}{ Time interval since screening, months } \\
\hline Never screened & 1.00 & Reference & 1.00 & Reference \\
\hline$\leqslant 11$ & 1.82 & $(1.69-1.96)$ & 1.91 & (1.75-2.09) \\
\hline $12-23$ & 1.48 & $(1.26-1.73)$ & 1.64 & $(1.36-1.97)$ \\
\hline $24-35$ & 1.53 & (1.13-2.07) & 1.50 & $(1.05-2.15)$ \\
\hline$\geqslant 36$ months or over & 1.38 & $(1.09-1.75)$ & 1.55 & $(1.18-2.04)$ \\
\hline \multicolumn{5}{|c|}{$\begin{array}{l}\text { Abbreviations: } \mathrm{Cl}=\text { confidence interval; } M A P=\text { medical aids programme; } \mathrm{NHI}=\text { national } \\
\text { health insurance; } \mathrm{OR}=\text { odds ratio; } \mathrm{UGIS}=\text { upper gastrointestinal series. } \\
{ }^{\mathrm{a}} \text { Analyses were conducted using conditional logistic regression model. } \\
{ }^{b} \text { Odds ratio of detecting localised gastric cancer in screened group vs never-screened } \\
\text { group in a subgroup }\end{array}$} \\
\hline
\end{tabular}

Nevertheless, this factor might have led to the underestimation of the magnitude of the observed screening effect. Finally, gastric cancer screening efficacy cannot be directly estimated by cancer stage, as there might be a gap related to lead-time bias and length bias between cancer stage and mortality. In this study, stage at diagnosis was used as an intermediate outcome measure to predict survival. However, lead-time bias occurs in such a way that the survival of screen-detected cases appears to be lengthened, whereas, in actuality, the screening simply advances the date of the diagnosis, extending the period between diagnosis and death, without actual prolongation of life. Also, a higher proportion of localised gastric cancers in the screening group might be associated with the tendency of screening to detect slow-growing lesions with better prognosis and to miss fast-growing lesions with poorer survival. Thus, the efficacy of any screening should be evaluated in terms of whether mortality from cancer is reduced in the screened population, and therefore, further study is needed to determine whether gastric cancer screening is effective in reducing mortality.

Despite of these limitations, this study suggests the following implications. First, gastric cancer screening using endoscopy was shown to be associated with an earlier stage at gastric cancer diagnosis in patients aged 40 years or over. Also, our data suggest that endoscopy is more likely to detect localised gastric cancer compared with UGIS. Although the data presented here are preliminary, our intermediate outcomes indicate that, in Korea, the introduction of gastric cancer screening by endoscopy in the average-risk population appears to perform better than screening by UGIS. However, data on the impact of endoscopy screening programmes on gastric cancer mortality are limited. Thus, further study is needed to determine whether endoscopy screening is more effective than radiography screening in reducing mortality.

\section{ACKNOWLEDGEMENTS}

This study was supported by a Grant-in-Aid for Cancer Research and Control from the National Cancer Center, Korea (Grant number: 1010200 and 1310231), and a grant from the National R\&D Programme for Cancer Control, Ministry of Health and Welfare, Republic of Korea (Grant number: 0820420).

\section{REFERENCES}

Cooper GS, Yuan Z, Chak A, Rimm AA (2002) Association of prediagnosis endoscopy with stage and survival in adenocarcinoma of the esophagus and gastric cardia. Cancer 95(1): 32-38.

Ferlay J, Soerjomataram I, Ervik M, Dikshit R, Eser S, Mathers C, Rebelo M, Parkin DM, Forman D, Bray F (2013) GLOBOCAN 2012 v1.0, Cancer Incidence and Mortality Worldwide: IARC CancerBase No. 11 [Internet]. available at http://globocan.iarc.fraccessed on 14 April 2013.

Fukao A, Tsubono Y, Tsuji I, HIsamichi S, Sugahara N, Takano A (1995) The evaluation of screening for gastric cancer in Miyagi Prefecture, Japan: a population-based case-control study. Int J Cancer 60(1): 45-48.

Hamashima C, Ogoshi K, Okamoto M, Shabana M, Kishimoto T, Fukao A (2013) A community-based, case-control study evaluating mortality reduction from gastric cancer by endoscopic screening in Japan. PLoS One 8(11): e79088.

Hosokawa O, Miyanaga T, Kaizaki Y, Hattori M, Dohden K, Ohta K, Itou Y, Aoyagi H (2008) Decreased death from gastric cancer by endoscopic screening: association with a population-based cancer registry. Scand J Gastroenterol 43(9): 1112-1115.

Jung KW, Won YJ, Kong HJ, Oh CM, Lee DH, Lee JS (2014) Cancer statistics in Korea: incidence, mortality, survival, and prevalence in 2011. Cancer Res Treat 46(2): 109-123.

Jung KW, Won YJ, Kong HJ, Oh CM, Shin A, Lee JS (2013) Survival of korean adult cancer patients by stage at diagnosis, 2006-2010: national cancer registry study. Cancer Res Treat 45(3): 162-171.

Karim-Kos HE, de Vries E, Soerjomataram I, Lemmens V, Siesling S, Coebergh JW (2008) Recent trends of cancer in Europe: a combined approach of incidence, survival and mortality for 17 cancer sites since the 1990s. Eur J Cancer 44(10): 1345-1389.

Kim Y, Jun JK, Choi KS, Lee HY, Park EC (2011) Overview of the National Cancer screening programme and the cancer screening status in Korea. Asian Pac J Cancer Prev 12(3): 725-730.

Leung WK, Wu MS, Kakugawa Y, Kim JJ, Yeoh KG, Goh KL, Wu KC, Wu DC, Sollano J, Kachintorn U, Gotoda T, Lin JT, You WC, Ng EK, Sung JJ. Asia Pacific Working Group on Gastric Cancer (2008) Screening for gastric cancer in Asia: current evidence and practice. Lancet Oncol 9(3): 279-287.

Miyamoto A, Kuriyama S, Nishino Y, Tsubono Y, Nakaya N, Ohmori K, Kurashima K, Shibuya D, Tsuji I (2007) Lower risk of death from gastric cancer among participants of gastric cancer screening in Japan: a population-based cohort study. Prev Med 44(1): 12-19.

Mizoue T, Yoshimura T, Tokui N, Hoshiyama Y, Yatsuya H, Sakata K, Kondo T, Kikuchi S, Toyoshima H, Hayakawa N, Tamakoshi A, Ohno Y, Fujino Y, Kaneko S. Japan Collaborative Cohort Study Group (2003) Prospective study of screening for stomach cancer in Japan. Int J Cancer 106(1): 103-107.

Mori Y, Arita T, Shimoda K, Yasuda K, Yoshida T, Kitano S (2001) Effect of periodic endoscopy for gastric cancer on early detection and improvement of survival. Gastric Cancer 4(3): 132-136.

Nam JH, Choi IJ, Cho SJ, Kim CG, Jun JK, Choi KS, Nam BH, Lee JH, Ryu KW, Kim YW (2012) Association of the interval between endoscopies with gastric cancer stage at diagnosis in a region of high prevalence. Cancer 118(20): 4953-4960. 
Nam SY, Choi IJ, Park KW, Kim CG, Lee JY, Kook MC, Lee JS, Park SR, Lee JH, Ryu KW, Kim YW (2009) Effect of repeated endoscopic screening on the incidence and treatment of gastric cancer in health screenees. Eur J Gastroenterol Hepatol 21(8): 855-860.

Nashimoto A, Akazawa K, Isobe Y, Miyashiro I, Katai H, Kodera Y, Tsujitani S, Seto Y, Furukawa H, Oda I, Ono H, Tanabe S, Kaminishi M (2013) Gastric cancer treated in 2002 in Japan: 2009 annual report of the JGCA nationwide registry. Gastric Cancer 16(1): 1-27.
Young J, Roffer S, Ries L, Fritz A, Hurlbutt A (2001) SEER Summary Stagingg manual - 2000: codes and coding instructions. National Cancer Institute: Bethesda, MD, USA, NIH Pub. no. 01-4969.

cc)(1)(2) This work is licensed under the Creative Commons cc) ${ }_{\mathrm{BY}} \mathrm{NC}$ SA Attribution-NonCommercial-Share Alike 3.0 Unported License. To view a copy of this license, visit http://creativecommons. org/licenses/by-nc-sa/3.0/ 